\title{
Morphological Development of Sambong (Blumea balsamifera (L.) DC.) Leaf Studied by Frozen Section and Thin Section
}

\author{
Liwei Liu1,2,3, Xiaolu Chen1,2, Quan Yang3, Yuxin Pang1,2* \\ ${ }^{1}$ Tropical Crops Genetic Resources Institute, Chinese Academy of Tropical Agricultural Sciences /Key Laboratory \\ of Crop Gene Resources and Germplasm Enhancement in Southern China, Ministry of Agriculture, Danzhou, \\ China \\ ${ }^{2}$ Hainan Provincial Engineering Research Center for Blumea balsamifera, Danzhou, China \\ ${ }^{3}$ School of Traditional Chinese Medicine, Guangdong Pharmaceutical University, Guangzhou, China \\ Email: "blumeachina@126.com
}

Received 3 March 2016; accepted 4 April 2016; published 7 April 2016

\begin{abstract}
The sambong (Blumea balsamifera (L.) DC.) leaf was investigated by applying frozen section and thin section technology, and observed through optical microscope. The results showed that the sambong leaf was firstly originated nearby the apical bud at the stem tip. The first leaf grows fast, that the mitosis happens frequently and the cytoplasm manufacture rapidly. The cells in the first few leaves have not yet differentiated into the spongy parenchyma and palisade parenchyma. In the mature leaf, the spongy parenchyma and palisade parenchyma were fully developed. The epidermal hairs were firstly developed on the abaxial side of the first leaf.
\end{abstract}

\section{Keywords}

\section{Sambong, Blumea balsamifera, Ainaxiang, Nalong, Morphological Development, Thin Section}

\section{Introduction}

Sambong (Blumea balsamifera (L.) DC.) is an ancient oriental medicinal herb, which grows in the wild in Southeast Asia. In China, Malaysia, Thailand, Vietnam, and Philippines, it has been used as a medicine to treat skin wounds, and as incense because it has a high level of essential oils [1] [2]. One of the most important extractions of sambong in China is the Traditional Chinese Medicine (TCM) Ai Pian, consisted of 85\% l-borneol [3]. Sambong oil, another extraction of the plant, is one of the ingredients of some nonprescription drugs (OTCs), for example, Jin Hou Jian Spray (Guizhou hongyu pharmaceutical co., LTD, China). It has been proved help skin wounds recover [4], and may be safe for liver when external applied [5].

Earlier studies showed that the leaf is the main source of Ai Pian and the sambong oil in sambong [6]. However, how the oil accumulated in leaf is yet unclear. As a result, studying the morphological development of

\footnotetext{
${ }^{*}$ Corresponding author.
} 
sambong leaf is necessary.

\section{Materials and Methods}

\subsection{Plant Materials}

Sambong plants were collected in Luodian (Guizhou, China), authenticated by Prof. Yu-Xin Pang. Voucher specimens (TCGRI 10011) have been deposited at the Traditional Medicinal Plant Germplasm Nursery of South China, Tropical Crops Genetic Resources Institute, Chinese Academy of Tropical Agricultural Sciences, Hainan, China.

\subsection{Frozen Section}

Fresh and healthy stems tips and leaves were collected. They were directly cut into $5 \mathrm{~mm}$ long or $4 \times 4 \mathrm{~mm}^{2}$ in area without fixed. Each pieces of stems tips or leaves were embedding on a sample table with Jung tissue freezing medium (Leica, German) under $-18^{\circ} \mathrm{C}$ for $10 \mathrm{~min}$, and then cut in Leica CM1900 under $-18^{\circ} \mathrm{C}$. The thickness range is $10-30 \mu \mathrm{m}$. The sections were observed with a computer-equipped microscope.

\subsection{Thin Section}

Fresh and healthy leaves were collected and cut into $1 \times 1 \mathrm{~mm}$ pieces. Fixed in $2.5 \%$ paraformaldehyde- $3.0 \%$ glutaraldehyde (dissolved in MPBS, $\mathrm{pH}$ 7.2) and kept under $4^{\circ} \mathrm{C}$ for $12 \mathrm{~h}$. Then wash the leaf pieces with phosphate buffer for three times. Post-fix the pieces with osmic acid under room temperature for $2 \mathrm{~h}$, then wash them with phosphate buffer for three times. Dehydration the pieces with 50\%, 70\%, 85\%, 95\%, 100\%, 100\% ethanol, then transition into epoxy propane. Embed the pieces with Epon812 epoxy resin. Kept them under $40^{\circ} \mathrm{C}$ for $24 \mathrm{~h}$, then $60^{\circ} \mathrm{C}$ for $24 \mathrm{~h}$. Cut the pieces with Leica EM UC6 microtome with a thickness of $900 \mathrm{~nm}$. Stain the sections with $0.5 \%$ toluidine blue. Observe the sections with a computer-equipped microscope.

\section{Results}

\subsection{Leaf Generation and Development}

The sambong leaf was firstly originated from leaf primordium nearby the stem tip. The first leaf grows fast, that the mitosis there happens frequently, and the cytoplasm manufacture rapidly (Figure 1(a) and Figure 1(b)). Midrib develops earlier than the blade (Figure 1(c)). The mesophyll cells in the first few leaves have not yet differentiated into the spongy parenchyma and palisade parenchyma (Figures 2(a)-(d)). Then the vascular tissue formed (Figure 2(e)). In the mature leaf, the spongy parenchyma and palisade parenchyma were fully developed (Figure 2(f)).

\subsection{Organs in Leaf Epidermis}

The stomas were firstly developed from the epidermis cells of the sambong leaf. There are two kinds of epidermal
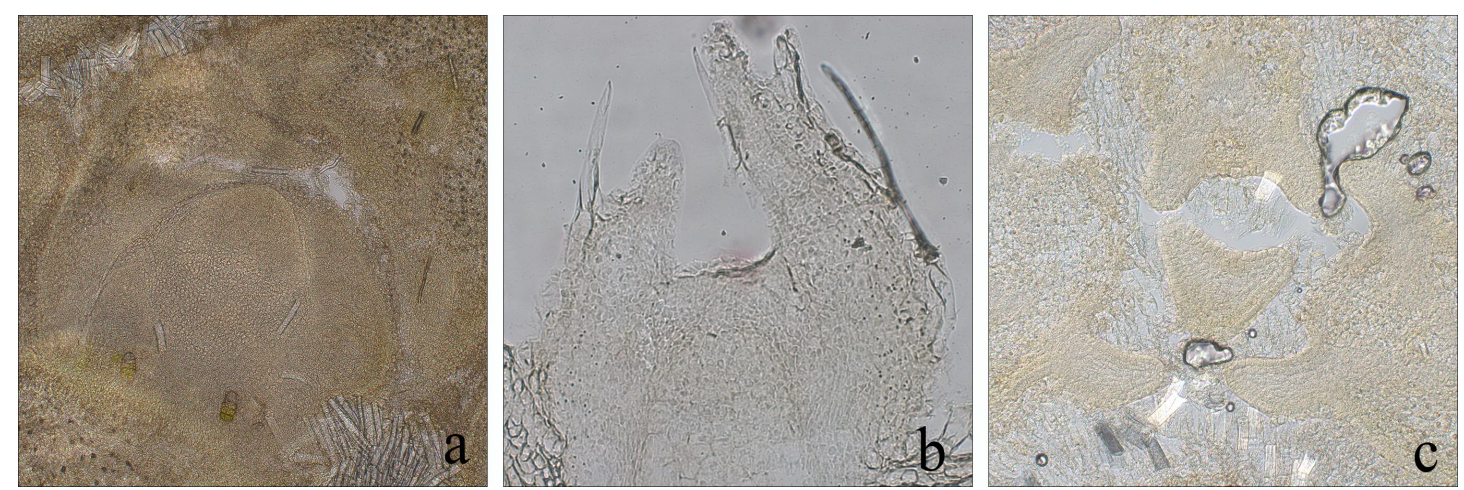

Figure 1. The morphological observation of sambong stem tip. (a) Shoot apical meristem transection; (b) Shoot apical meristem axial section; (c) The first euphylla transaction. 

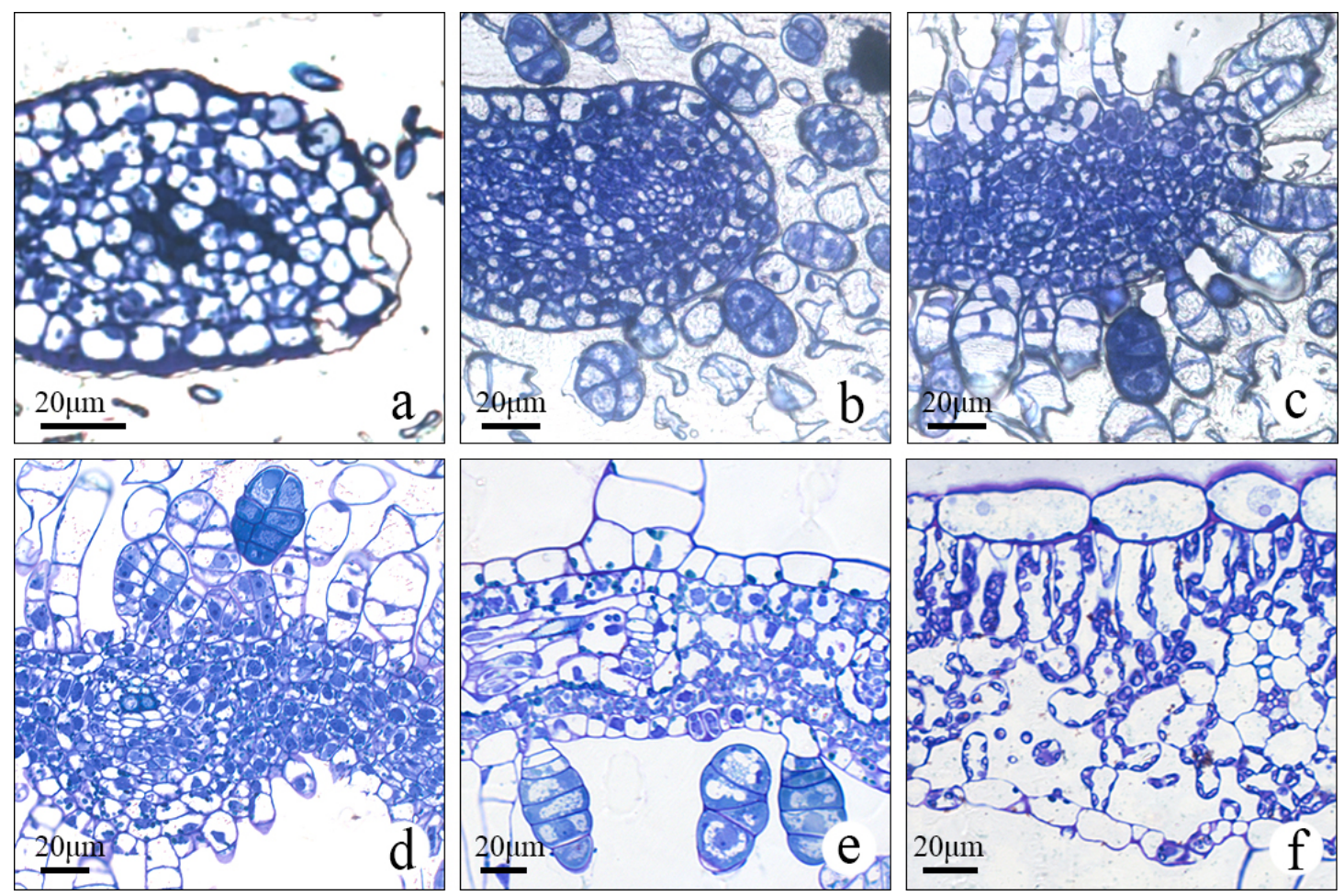

Figure 2. The morphological development of sambong leaf. (a) Apex of a leaf; (b) Leaf primordium; (c) Apex of a young leaf; (d)-(f) Leaf with different development stages.

hairs in sambong leaf epidermis. The non-glandular hairs do not secrete. These hairs are usually long, consists of multiple cells with thin cytoplasm. The glandular hairs secrete. They are much shorter than the non- glandular hairs, usually 40 - $60 \mu \mathrm{m}$ by length, consists of two columns of cells with dense cytoplasm (Figure 2(d)). Most epidermal hairs generate from the epidermis cells on the first leaf. Less epidermis hairs were found generated on mature leaf. As the leaf grow, the density of the epidermal hairs decrease (Figures 2(d)-(f)).

\subsection{Structure of Mature Leaf}

The mature sambong leaf is bifacial leaf. Leaf epidermis is consisted of one layer of cells. The upper epidermis is thicker than the lower one. There were stomata on the upper and the lower epidermis while the stomata frequency in lower epidermis is higher. The mesophyll tissue in leaf was developed into palisade parenchyma and spongy parenchyma. The former is nearby the upper epidermis, and is consisted by a layer of columnar cells. The latter, loose irregularly shaped cells, packed with large air spaces, allow rapid diffusion of gases to.

\section{Conclusions}

The basic microstructure of mature sambong leaf observed in present study was similar with those observed in previously researches. But very few information can be found on the developmental morphology of this plant.

Results in present study shows that the sambong leaf was firstly originated nearby the apical bud at the stem tip. The epidermal hairs were firstly developed on the abaxial side of the first euphylla. It's much earlier than the development of the spongy parenchyma and palisade parenchyma. However, the firstly developed hairs were not glandular hairs. We also observed no oil cells, oil chambers, or vitta in the young leaves and stem tips. As a result, we preliminarily consider there was no special structure of essential oil secretion in young leaves and stems. The oil maybe not accumulated in those first few leaves and stems by special structure. The further study should be focus on locating the oil in the leaf by using staining the oil.

\section{Acknowledgements}

This study was funded by the Fundamental Scientific Research Funds for CATAS-TCGRI, the Ministry of 
Agriculture (\#1630032015035), and the Natural Science of Foundation of Hainan Province, China (\#20153074).

\section{References}

[1] Pang, Y.X., Wang, D., Fan, Z.W., Chen, X., Yu, F., Hu, X., Wang, K. and Yuan, L. (2014) Blumea balsamifera-A Phytochemical and Pharmacological Review. Molecules, 19, 9453-9477. http://dx.doi.org/10.3390/molecules19079453

[2] Chen, M. (2009) Studies on the Active Constituents of Blumea balsamifera. Master Thesis, Shanghai Jiao Tong University, Shanghai.

[3] Chinese Pharmacopeia Commission, Ed. (2015) Pharmacopoeia of the People’s Republic of China. 2010 ed. ed. The Medicine Science and Technology Press of China, Beijing.

[4] Pang, Y.X., Wang, D., Hu, X., Wang, H., Fu, W.J., Fan, Z.W., Chen, X.L. and Yu, F.L. (2014) Effect of Volatile oil from Blumea Balsamifera (L.) DC. Leaves on Wound Healing in Mice. Journal of Traditional Chinese Medicine, 6, 716-724. http://dx.doi.org/10.1016/S0254-6272(15)30087-X

[5] Pang, Y.X., Fan, Z.W., Wang, D., Yang, Q., Wang, K., Chen, X.L., Hu, X., Yu, F.L. and Chen, Z.X. (2014) External Application of the Volatile Oil from Blumea balsamifera May Be Safe for Liver-A Study on Its Chemical Composition and Hepatotoxicity. Molecules, 19, 18479-18492. http://dx.doi.org/10.3390/molecules191118479

[6] Wang, Y.H., Tian, H.Y., He, S.J., Hu, Q.P., Wang, H.X., Zhou, C.L. and Wang, X. (2012) Analysis of Volatile Components from Leaf in Blumea balsamifera (L.) DC. with Different Extraction Methods by Gas Chromatography-Mass Spectrometry. Science and Technology of Food Industry, 33, 97-105. 